Article

\title{
The Effects of a Chactoid Scorpion Venom and Its Purified Toxins on Rat Blood Pressure and Mast Cells Histamine Release
}

\author{
Keren Ettinger, Gadi Cohen, Tatjana Momic and Philip Lazarovici *
}

Institute for Drug Research, School of Pharmacy, Faculty of Medicine, The Hebrew University of Jerusalem, POB 12065, Jerusalem 91120, Israel; E-Mails: keren.ettinger@gmail.com (K.E.); gadifco@gmail.com (G.C.); momict@gmail.com (T.M.)

* Author to whom correspondence should be addressed; E-Mail: philip1@ekmd.huji.ac.il; Tel.: +972-2-6758729; Fax: +972-2-6757490.

Received: 23 May 2013; in revised form: 25 June 2013 / Accepted: 18 July 2013 /

Published: 29 July 2013

\begin{abstract}
The effect of the venom of the Chactoid family of scorpions on blood pressure was scantly investigated and was addressed in the present study using the venom of the Israeli scorpion, Scorpio maurus palmatus. Blood pressure in rats was monitored via cannulated femoral artery, while venom and toxins were introduced into femoral vein. Venom injection elicited a biphasic effect, expressed first by a fast and transient hypotensive response, which lasted up to $10 \mathrm{~min}$, followed by a hypertensive response, which lasted up to one hour. It was found that these effects resulted from different venom components. Phospholipase $\mathrm{A}_{2}$ produced the hypotensive effect, while a non-enzymatic neurotoxic polypeptide fraction produced the hypertensive effect. Surprisingly, the main neurotoxic polypeptide to mice had no effect on blood pressure. In vitro experiments indicated that the hypertensive factors caused histamine release from the peritoneal mast cells, but this effect is assumed to be not relevant to their in vivo effect. In spite of the cytotoxic activity of phospholipase $\mathrm{A}_{2}$, it did not release histamine. These findings suggest that the effects of venom and isolated fractions on blood pressure parameters are mediated by different mechanisms, which deserve further pharmacological investigation.
\end{abstract}

Keywords: chactoid scorpion; Scorpio maurus palmatus; venom; phospholipase $\mathrm{A}_{2}$; neurotoxin; blood pressure; histamine release 


\section{Introduction}

Scorpions are venomous arthropods, members of the Arachnida class and the order, Scorpiones. They are found in all continents, including Israel. The scorpion species that are medically important in Israel belong to the families of Buthidae and Scorpionidae, represented by the genera, Leiurus quinquestriatus quinquestriatus and Scorpio maurus palmatus. The envenomation by these scorpions is considered a public and veterinary health problem in Israel, as in other countries [1]. The clinical symptoms upon envenomation indicate a general stimulation of the autonomic, somatic and peripheral nervous systems [2]. The severity of scorpion envenomation and the increased risk of mortality, especially among children, is mainly attributed to cardio-respiratory pathology, therefore stimulating investigations on the effect of scorpion venoms and derived toxins on the hemodynamic perturbations in different animal models [3-6].

Earlier studies indicated that the venom of Scorpio maurus palmatus consists of three main groups of polypeptides: neurotoxins, which inhibit neuronal ionic channel conductance [7,8], phospholipases $\mathrm{A}_{2}$ [9] and cytotoxins [7]. The toxicity of the venom results from synergistic interactions between these groups of components $[9,10]$. However, the effect of venom and isolated compound on the blood pressure was not yet investigated and, therefore, addressed in the present study in a rat model.

\section{Results}

\subsection{The Effect of Scorpio maurus palmatus Venom on Blood Pressure}

Low doses of the chactoid scorpion venom injected intravenously elevated the mean arterial blood pressure from $60 \pm 5$ to $80 \pm 5 \mathrm{~mm} \mathrm{Hg}(n=18 ; p<0.05)$ (Figure 1, upper part). This hypertensive effect was dose-dependent over the doses of $0.01-0.10 \mathrm{mg} / \mathrm{kg}(n=9$ for each dose), continued for some 50-80 min and the blood pressure returned to its control values. At higher doses $(0.2 \mathrm{mg} / \mathrm{kg})$, the venom first elicited a short hypotensive effect, which lasted 5-7 min, and then, a hypertensive effect was observed $(n=9)$ (Figure 1, middle part) similar to the lower dose effect. Such a biphasic action was also seen following injection of a lethal dose $(0.4 \mathrm{mg} / \mathrm{kg} ; n=6)$. Interestingly, the blood pressure returned to control values $(60 \mathrm{~mm} \mathrm{Hg}$ ), then a second and violent burst of hypersensitivity (up to $120 \mathrm{~mm} \mathrm{Hg}$ ), which lasted approximately $10 \mathrm{~min}$, occurred. During these events, skeletal muscle convulsions accompanied with sporadic respiratory apneas and heart arrhythmias appeared, then the blood pressure dropped and animal death occurred (Figure 1, lower part).

\subsection{Chromatographic Separation of the Venom and Identification of Fractions Responsible for Blood Pressure Perturbation}

In order to identify the venom proteins responsible for the perturbation of the blood pressure, the Scorpio maurus palmatus (Smp) venom was separated by column chromatography on Sephadex G-50 gel (Figure 2) [7,9]. This chromatographic separation generated several protein fractions: Smp1, Smp2, Smp3, Smp4 and Smp5. Phospholipase $\mathrm{A}_{2}\left(\mathrm{PlA}_{2}\right)$ was detected in the Smp2 fraction, while neurotoxicity to mice and rats was detected in the Smp4 fraction. The Smp3 fraction was cytotoxic to 
cell cultures of sympathetic neurons (PC12 cells) and hemolytic to human red blood cells (data not shown) (Figure 2).

Figure 1. The typical effect of Scorpio maurus palmatus venom on blood pressure. The original tracing of the experiments show the blood pressure response $(\mathrm{mm} \mathrm{Hg})$ after addition of different doses of venom indicated by the vertical arrow. Each trace represents a single rat receiving a single dose of venom (representing measurements of eight rats). The length of the horizontal lines represents the time course of the experiment: thin line (sec); thick line (min).

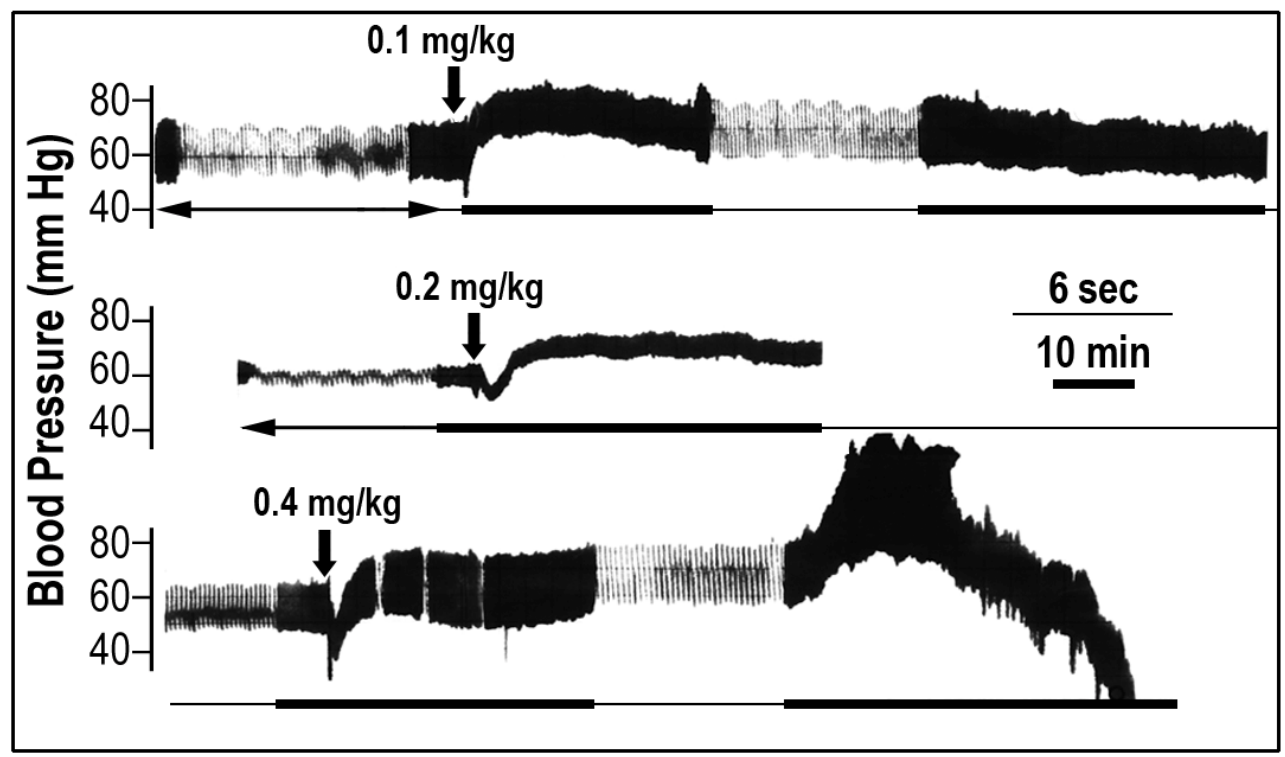

Figure 2. Gel filtration chromatography of Scorpio maurus palmatus venom. $500 \mathrm{mg}$ of venom were separated on Sephadex G-50 gel equilibrated and eluted by $0.1 \mathrm{M}$ ammonium acetate, $\mathrm{pH}$ 8.5. The flow rate was $15 \mathrm{~mL} / \mathrm{h}$, and fractions of $10 \mathrm{~mL}$ were collected. The marked areas correspond to $\mathrm{PlA}_{2}$ and neurotoxic fractions, respectively. Full circles represent protein absorbance at $280 \mathrm{~nm}$, and open circles correspond to the $\mathrm{PlA}_{2}$ activity.

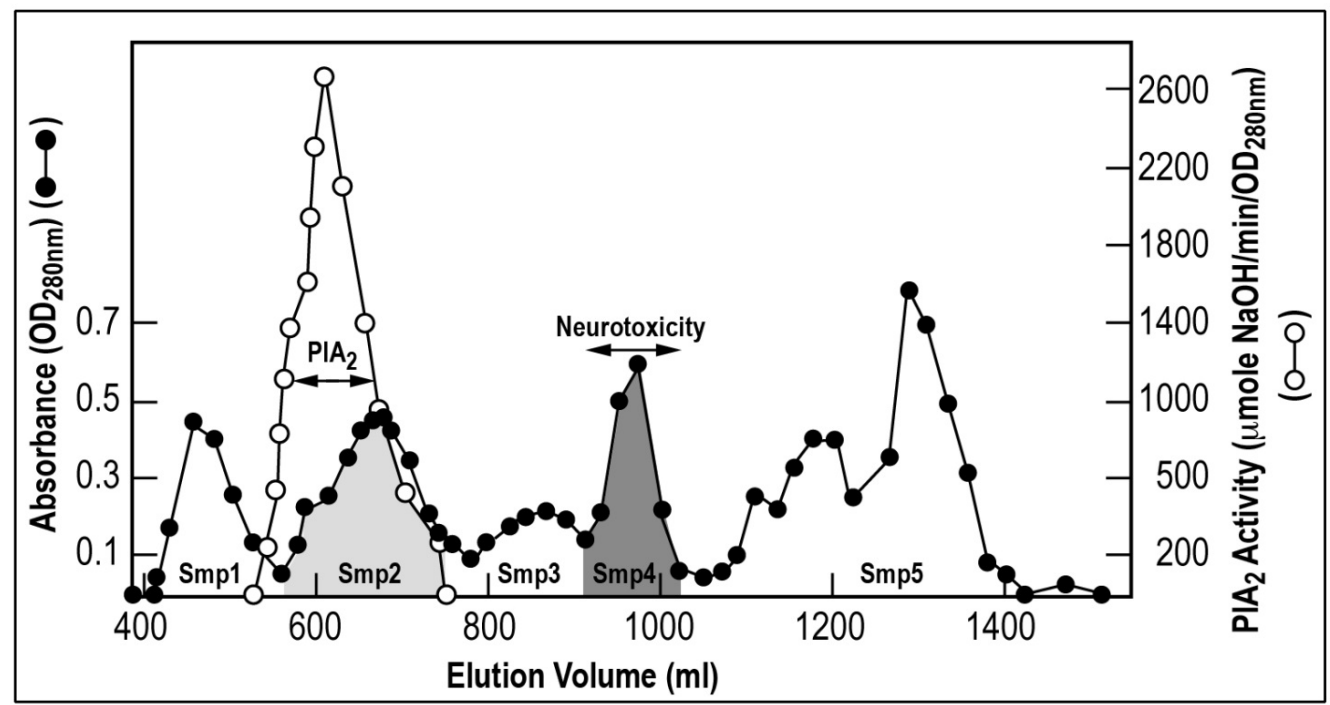


In the next step, we evaluated the effects of the isolated fractions on the arterial blood pressure of the rats (Figure 3). Fractionation of the venom revealed that the hypertensive and hypotensive effects resulted from different venom protein fractions. The hypertensive activity was attributed to the neurotoxic fraction, Smp4 (Figure 3A-a). This fraction not only increased the blood pressure, but also caused respiratory problems, mimicking the whole venom effect. In the past, several toxins were isolated from this fraction [9], and therefore, we used them to further investigate their potential hypertensive activity. While CT3a neurotoxin at a concentration of $0.4 \mathrm{mg} / \mathrm{kg}$ elicited a clear hypertensive effect (Figure 3A-b), which spontaneously returned to normal value after $90 \mathrm{~min}$, the neurotoxin, CT3b, elicited respiratory perturbations with instability of animal blood pressure (Figure 3A-C). The neurotoxin for mice, named mammal toxin (Smp MT) [9], and the toxin to insects (Smp IT) were found ineffective in perturbation of rat blood pressure up to high doses of $1 \mathrm{mg} / \mathrm{kg}$ (Figure 3A-d,e), although for Smp MT, symptoms of muscle paralysis were observed. The hypotensive response of the venom was only reproduced by the $\mathrm{PlA}_{2}$ containing fraction at a dose of $0.07 \mathrm{Smp} 2$ (Figure 3B-a) and at $0.1 \mathrm{mg} / \mathrm{kg}$ for the purified $\mathrm{PlA}_{2}-\mathrm{A} 1 \mathrm{~b}$ enzyme (Figure 3B-b) [9], respectively. The Smp3 fraction, characterized by cytotoxic and cytolytic activity, was not effective in modulating blood pressure up to $1 \mathrm{mg} / \mathrm{kg}$.

Figure 3. The effect on blood pressure of Scorpio maurus palmatus venom protein fractions isolated by gel permeation and purified toxins. (A) Neurotoxins effect on blood pressure; (B) $\mathrm{PlA}_{2}$ effect on blood pressure; (C) reconstitution of whole venom effect and synergistic effect on blood pressure of mixed doses of neurotoxin and $\mathrm{PlA}_{2}$ at different protein ratios. Each trace represents a single rat receiving a single dose of tested compound (representing measurements of 6-8 rats). The length of the horizontal lines represents the time course of the experiment: thin line (sec); thick line (min).

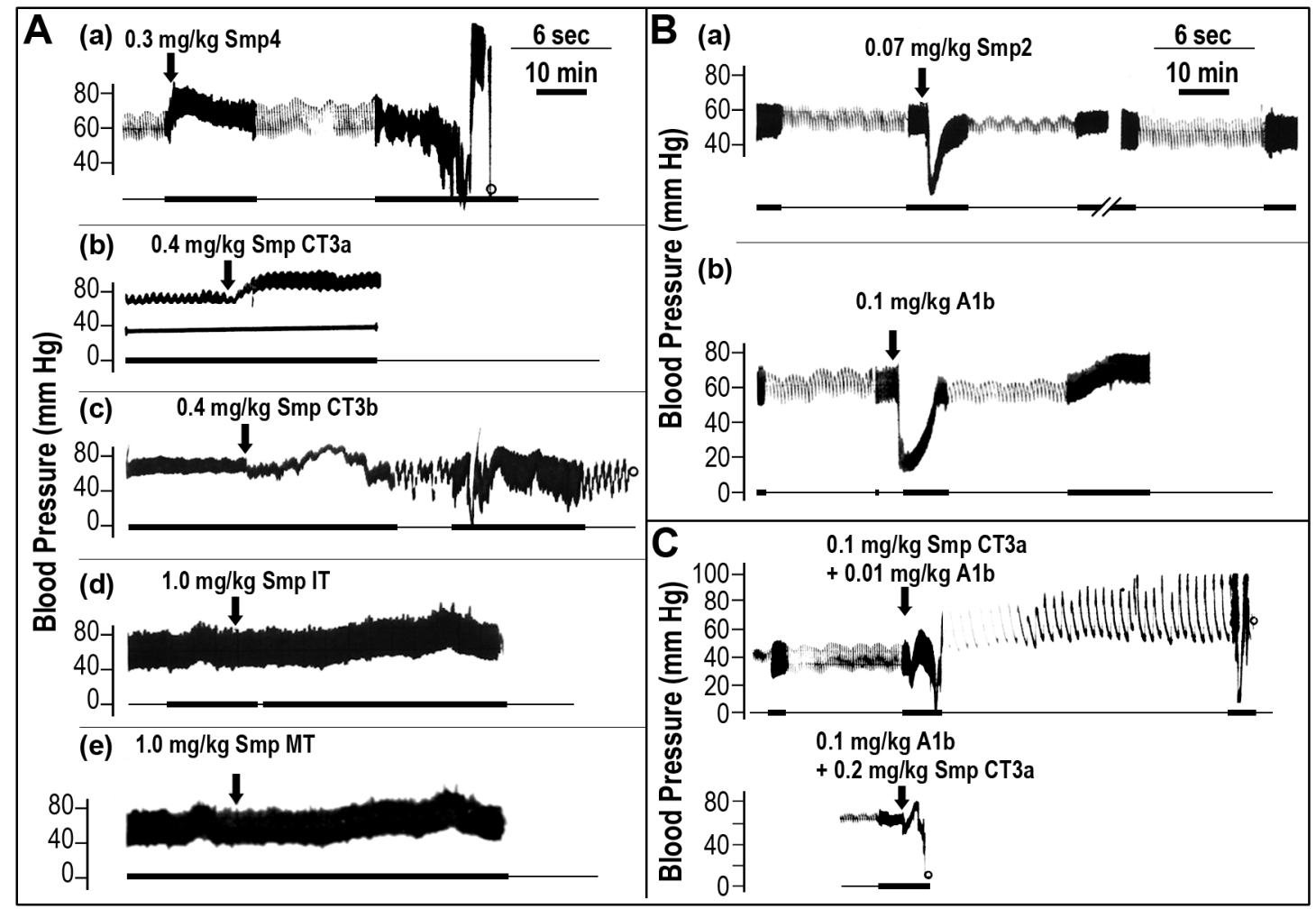


It was shown previously that the addition of the purified A1b-P1A $\mathrm{A}_{2}$ enzyme elevated the lethal potency of the neurotoxins [9]. Since the Scorpio maurus palmatus venom biphasic effect on blood pressure is due to both the $\mathrm{PlA}_{2}$ and neurotoxin fractions, we tested qualitatively whether a combination of the purified A1b-PlA $\mathrm{A}_{2}$ enzyme and Smp CT3a neurotoxin can mimic the dual effect of the venom on blood pressure, upon concomitant injection at a non-toxic, very low dose. While injected alone at low doses, the purified $\mathrm{A} 1 \mathrm{~b}-\mathrm{PlA}_{2}$ enzyme $(0.01 \mathrm{mg} / \mathrm{kg})$ and the hypertensive factor CT3a $(0.1 \mathrm{mg} / \mathrm{kg})$ were found non-toxic and did not alter the blood pressure parameters. However, injection of their mixture at similar very low doses elicited a biphasic effect on blood pressure, followed by respiratory problems and animal death, mimicking the effect of the whole venom (Figure 3C-upper part). This effect was also observed at a higher dose ratio of $\mathrm{A} 1 \mathrm{~b}-\mathrm{PlA}_{2}$ enzyme and the hypertensive factor, CT3a (Figure 3C-lower part).

\subsection{The Effect of Venom and Isolated Protein Fractions on Histamine Release from Mast Cells}

Using the in vitro assay of histamine release from peritoneal mast cells, we evaluated the ability of Scorpio maurus palmatus venom and isolated fractions to release histamine by comparison to compound 48/80. Figure 4 clearly indicates that the Scorpio maurus palmatus whole venom induced histamine release in a dose-dependent manner, the effect of $50 \mu \mathrm{g} / \mathrm{mL}$ having been equivalent to that of a dose of $0.5 \mu \mathrm{g} / \mathrm{mL}$ of compound 48/80. Microscopic observation of the cells upon venom treatment, revealed typical degranulation and an increase in membrane permeability, as evidenced by the uptake of trypan blue (data not shown).

The fraction Smp2 and derived $\mathrm{A} 1 \mathrm{~b}-\mathrm{PlA}_{2}$ have shown only a very low effect on histamine release. Smp4 fraction and derived neurotoxins, CT3a, CT3b and MT, at $200 \mu \mathrm{g} / \mathrm{mL}$ caused histamine release similar to a dose of $1 \mu \mathrm{g} / \mathrm{mL}$ of compound $48 / 80$ and with a higher specific activity than the whole venom.

These results indicate that the histamine-induced released effect of the whole venom is produced in vitro by the Smp4 neurotoxic fraction and derived neurotoxins.

Figure 4. The effect of Scorpio maurus palmatus venom and protein fractions isolated by gel permeation and purified toxins on histamine release in vitro from mast cells.

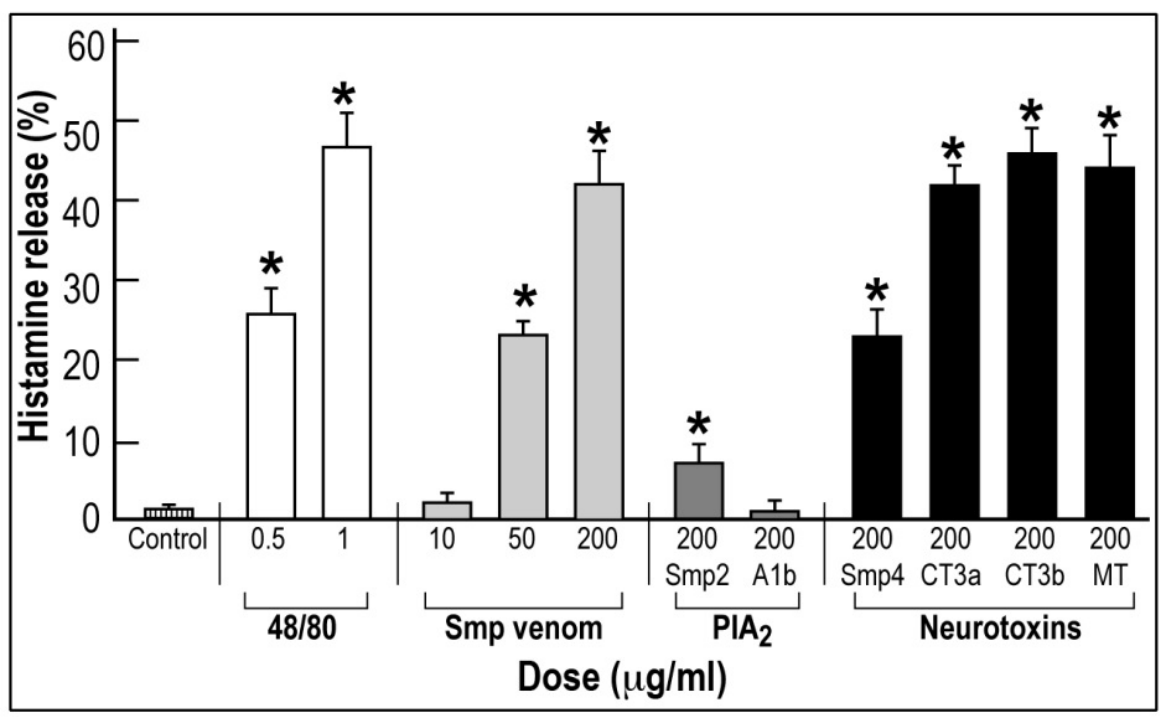




\subsection{Discussion}

The present study describes the complex effect of Scorpio maurus palmatus venom on rat blood pressure and identifies the hypertensive and hypotensive factors responsible for the biphasic effect of the whole venom. The present study unambiguously identified the phospholipase $\mathrm{A}_{2}$ protein fraction, Smp2, and derived phospholipase A1b [9] as responsible for this hypotensive effect. Phospholipases $\mathrm{A}_{2}$ were not found in Buthidae venoms; however, they are present in many snake venoms, which induces similar transient hypotensive effects on blood pressure [11]. The suggested mechanism of their action involves production of vasodilators and activation of a cyclooxygenase-dependent system [12,13]. Interestingly, from the point of view of scorpion venom pharmacology, the Scorpio maurus palmatus venom evolution regarding hemodynamics effects on blood pressure resembles evolutionary aspects of snake's venoms. Since it has been reported that scorpion venoms induce mast cell degranulation and histamine release [14,15] and since histamine by activating $\mathrm{H} 1$ histamine receptor on endothelial cells [16] and generating nitric oxide produces vasodilation and fast hypotensive effects, we sought to investigate if the hypotensive effect of Scorpio maurus palmatus venom and isolated $\mathrm{PlA}_{2}$ is mediated by histamine release in vitro (Figure 4) and in vivo (data not shown). Indeed, the Scorpio maurus palmatus venom similar to the reference compound 48/80 induced a dose-dependent degranulation and histamine release from mast cells. Surprisingly, this effect was not mediated by $\mathrm{PlA}_{2}$, but was induced by all neurotoxins originally isolated from the neurotoxin fraction, Smp4. This effect in vitro may be attributed to the high isoelectric point of these toxins inducing nonspecific in vitro degranulation and histamine release from mast cells [17]. Furthermore, this effect of Smp4 neurotoxic fraction observed in vitro could not be reproduced in vivo, since no histamine was released in the blood of rats injected with Smp4 fraction at a dose of 200-500 $\mu \mathrm{g} / \mathrm{kg}$ (data not shown). Therefore, we may hypothesize that the histamine induced release in vitro by neurotoxins is not relevant for the in vivo effects on blood pressure, and most probably, the venom effect is mediated in vivo by other unknown factors/mechanisms requiring future identification.

Our study also clearly identifies the neurotoxic Smp4 fraction as responsible for the hypertensive effect of the whole venom. This fraction contains several very basic and low molecular weight (31-34 amino acids) polypeptides, which were found to be relatively neurotoxic to different groups of animals, such as insects, crustacean and mammals [7-9]. From this series of neurotoxins, only CT3a and CT3b, previously defined as neurotoxins affecting crustacean [9], produced a clear hypertensive effect. Surprisingly, the other neurotoxins, including the mammal toxin, did not affect rat blood pressure (Figure 3). It is well established that mammal toxins from Buthidae venom interact with ionic channels of excitable cells, leading to a massive release of neurotransmitters. Voltage-gated $\mathrm{Na}+$ channel toxins are mainly responsible for the toxic effects of scorpion envenomation and hypertensive effect on blood pressure $[18,19]$. The structure of the Scorpio maurus palmatus neurotoxins is unknown and, therefore, cannot be classified yet to either $\alpha$ or $\beta$ scorpion toxins [18]. However, their similarity in amino acid composition to Buthidae mammal toxins [8] and ability to induce flaccid paralysis in albino mice upon cranial intraventricular injection at doses of 150-300 ng/kg, resembling the effects of Tityus serrulatus $\beta$ scorpion toxin, strongly suggest that Smp neurotoxins CT3a and CT3b may also induce the hypertensive effect by activation of the voltage-sensitive sodium channel followed by catecholamine release, responsible for the peripheral blood vessel vasoconstrictor effect 
and the increase in blood pressure. Furthermore, CT3a polypeptide acted cooperatively with the A1b phospholipase, at nontoxic concentrations, mimicking the venom effect with respect to the biphasic modulation on blood pressure and lethality (Figure 3C). These findings may suggest that the previously noticed synergistic effect of Scorpio maurus palmatus venom toxins [7,9] is also reproduced at the level of the complex hemodynamic interaction between the venom components on blood pressure.

The regulation of blood pressure is mediated by heart activity, kidney, adrenal, lung, vascular system, baroreceptors, brain medulla cardiovascular centers and blood biochemistry. Therefore, in order to pinpoint the precise mechanisms by which Scorpio maurus palmatus venom and derived toxins are perturbing the blood pressure is very important to first study their effects on these different physiological systems before agreeing on a pharmacological mechanism contributing to their complex effect on blood pressure. Clinicians dealing with the stabilization of the blood pressure in the envenomed patient, in particular children, advocate administration of antiserum therapy [6]. Since the Scorpio maurus palmatus venom effect on the blood pressure is very fast, before most patients are brought to a medical facility, and since the pharmacokinetics of this venom components are unknown and no serotherapies are available, the only way to stabilize the hemodynamic failure following Scorpio maurus palmatus envenomation is a pharmacological approach employing conventional autonomic drugs, such as dobutamine [4], clonidine [20], prazosin [21] and other drugs, according to the time course of appearance and type of blood pressure pathophysiology.

\section{Experimental Section}

\subsection{Materials}

The Scorpio maurus palmatus venom was obtained by "electrical milking" of field-collected scorpions and stored lyophilized until use [8]. Histamine and histamine releasing factor 48/50 were purchased from Sigma (St. Louis, USA).

\subsection{Venom Separation by Gel Filtration}

The extraction of the venom of the scorpion Scorpio maurus palmatus and the column chromatography procedures were used as previously reported [7,9]. Fractions with phospholipase $\mathrm{A}_{2}$ activity (Smp2 and A1b) and neurotoxins to different groups of animals (Smp4), crustacean toxins (CT) and a mammal toxin (MT) were obtained according to Lazarovici et al. [7,9] and Lazarovici and Zlotkin [8].

\subsection{Blood Pressure Measurement}

Adult rats of the Wistar strain (200-250 g) were used for these experiments. The rats had free access to water and standard chow until the day of the experiment. Experiments and animal care were approved by the Committee of Ethics of The Hebrew University and were performed in strict accordance with the Guide for the Care and Use of Laboratory Animals published by the US National Institutes of Health. Repeated administration of anesthesia was induced with pentobarbital sodium $(50 \mathrm{mg} / \mathrm{kg}$ ) intraperitoneally (i.p.), followed by tracheotomy and artificial ventilation, and body 
temperature was kept at $37-38{ }^{\circ} \mathrm{C}$ by a thermostatically controlled heating pad. Animals were ventilated with room air at 35-45 breaths/min supplemented with $\mathrm{O}_{2}$. At the end of the experiments, we confirmed that the arterial blood $\mathrm{pH}, \mathrm{pCO}_{2}$ and bicarbonate were within physiological ranges using an ABL 80 Flex-pH/Blood Gas Analyzer. For blood pressure recording, a femoral artery was cannulated and connected to a Narco Bio-System, RP-1500 pressure transducer. A Narco Bio-System DMP-4B physiograph was used to record blood pressure. The tested substances were injected in a single dose per rat, and experiments were repeated in 8 rats $(n=8)$ in a volume of $0.2-0.4 \mathrm{~mL}$ in heparinized saline via the cannulated femoral vein. The mean arterial pressure was calculated using the following formula: (diastolic $+($ systolic - diastolic) $/ 3)$. The results presented are from separate rats.

\subsection{Histamine Release Assay}

Mast cells were collected from the peritoneal perfusion with Tyrode from male inbred Wistar rats (200-300 g). Aliquots of $0.5 \mathrm{~mL}$ of $5 \times 10^{4}$ peritoneal mast cell suspensions were equilibrated at $37{ }^{\circ} \mathrm{C}$ in Tyrode solution $\left(137 \mathrm{mM} \mathrm{NaCl}, 2.7 \mathrm{mM} \mathrm{KCl}, 0.4 \mathrm{mM} \mathrm{NaH}_{2} \mathrm{PO}_{4}, 1.4 \mathrm{mM} \mathrm{CaCl}_{2}, 10 \mathrm{mM}\right.$ Hepes, $5.6 \mathrm{mM}$ glucose, $\mathrm{pH}$ 7.4). Half milliliter of the tested compound dissolved in Tyrode and equilibrated at $37{ }^{\circ} \mathrm{C}$ was added to the above cell suspension, and incubation was carried for $30 \mathrm{~min}$ at $37{ }^{\circ} \mathrm{C}$. The reaction was terminated by adding $1.5 \mathrm{~mL}$ of ice-cold calcium free Tyrode solution. The cells were isolated by centrifugation at $1,000 \times \mathrm{g}$ for $5 \mathrm{~min}$ and the supernatants collected. The pellets were resuspended in $2.5 \mathrm{~mL}$ Tyrode solution, placed for $10 \mathrm{~min}$ in a boiling water bath to release their residual histamine and centrifuged at $1,000 \times g$ for $5 \mathrm{~min}$. Aliquots from these supernatants were also collected. Histamine was assayed fluorometrically according to the method of Shore et al. [22]. Histamine release was calculated as a percentage of the total histamine content in each sample and subtracting the histamine released spontaneously (not exceeding 7\%) [23]. Lytic damage and cell degranulation was confirmed by microscopic observation and trypan blue staining [10].

\subsection{Phospholipase $A_{2}$ Activity Measurement}

Phospholipase activity was determined by a potentiometric titration $(0.1 \mathrm{M} \mathrm{NaOH})$ of acidity resulting from the release of fatty acids from a $1 \%$ suspension of phosphatidylcholine in the presence of $0.1 \%$ deoxycholate and $0.05 \mathrm{M} \mathrm{CaCl}_{2}$ at $\mathrm{pH} 8.0$ and $37{ }^{\circ} \mathrm{C}$, according to established methods [9]. The initial reaction velocity was determined, and the activity unit was defined as the amount of enzyme preparation releasing $1 \mu \mathrm{mol}$ of fatty acids per minute.

\subsection{Toxicity}

Toxicity to albino mice $(12-20 \mathrm{~g})$ on arthropods and insects was determined by subcutaneous injection, as previously described [7-9].

\subsection{Statistics}

The blood pressure results are presented as the mean \pm SD of three to six independent experiments ( $n=6-18$ rats) and were evaluated using the InStat3 statistics program (GraphPad, La Jolla, CA, USA). The histamine release results are presented as the mean $\pm \mathrm{SD}$ of three independent experiments 
$(n=12)$. Statistically significant differences between experimental groups were determined by the Student's $t$-test and ANOVA, followed by the Bonferroni post-test and were considered significant when $p \leq 0.05$.

\section{Conclusions}

Scorpio maurus palmatus venom upon injection into the femoral vein of anesthetized rats produced complex effects on the blood pressure. Using chromatographic separation of the venom and monitoring the femoral arterial pressure upon injection of the isolated proteins, it has been found that the hypotensive effect of the venom is caused by the $\mathrm{PlA}_{2}$ fraction and derived purified enzyme, while the hypertensive effect is caused by the neurotoxic fraction and purified crustacean neurotoxins. The whole venom effect on blood pressure was mimicked by mixing samples of the isolated hypotensive and hypertensive factors. The pharmacological mechanisms responsible for these effects are yet unknown and deserve clarification in order to design a therapeutic strategy for stabilization of the blood pressure in the envenomed patients.

\section{Acknowledgments}

P.L. holds Jacob Gitlin chair in physiology and is affiliated with and acknowledges the financial support of David R. Bloom Center of Pharmacy and Dr. Adolf and Klara Brettler Center for Research in Molecular Pharmacology and Therapeutics at The Hebrew University of Jerusalem, Israel. We would like to acknowledge Zehava Cohen for the help with the graphics.

\section{Conflict of Interest}

The authors declare no conflict of interest.

\section{References}

1. Chippaux, J.P.; Goyffon, M. Epidemiology of scorpionism: A global appraisal. Acta Trop. 2008, 107, 71-79.

2. Petricevich, V.L. Scorpion venom and the inflammatory response. Mediat. Inflamm. 2010, 2010, 903295.

3. Abdoon, N.A.; Fatani, A.J. Correlation between blood pressure, cytokines and nitric oxide in conscious rabbits injected with Leiurus quinquestriatus quinquestriatus scorpion venom. Toxicon 2009, 54, 471-480.

4. Hakim, A.; Sahnoun, Z.; Kallel, H.; Kassis, M.; Bouaziz, M.; Zeghal, K.M. Beneficial effects of high dobutamine doses on hemodynamic perturbations induced by Buthus occitanus tunetanus venom in rats. Med. Sci. Monit. 2009, 15, BR71-BR74.

5. Nouira, S.; Elatrous, S.; Besbes, L.; Boukef, R.; Devaux, C.; Aubrey, N.; Elayeb, M.; Abroug, F. Neurohormonal activation in severe scorpion envenomation: Correlation with hemodynamics and circulating toxin. Toxicol. Appl. Pharmacol. 2005, 208, 111-116. 
6. Tarasiuk, A.; Khvatskin, S.; Sofer, S. Effects of antivenom serotherapy on hemodynamic pathophysiology in dogs injected with L. quinquestriatus scorpion venom. Toxicon 1998, 36, 963-971.

7. Lazarovici, P.; Yanai, P.; Pelhate, M.; Zlotkin, E. Insect toxic component from the venom of a chactoid scorpion, Scorpio maurus palmatus (Scorpionidae). J. Biol. Chem. 1982, 257, 8397-8404.

8. Lazarovici, P.; Zlotkin, E. A mammal toxin derived from the venom of a chactoid scorpion. Comp. Biochem. Physiol. C 1982, 71, 177-181.

9. Lazarovici, P.; Menashe, M.; Zlotkin, E. Toxicity to crustacea due to polypeptide-phospholipase interaction in the venom of a chactoid scorpion. Arch. Biochem. Biophys. 1984, 229, 270-286.

10. Lazarovici, P.; Menashe, M.; Primor, N.; Hochman, J.; Zlotkin, E. The cytotoxicity of a cobra venom phospholipase to mouse lymphoma cells. Arch. Toxicol. 1982, 51, 167-173.

11. Lee, C.Y. Mode of Action of Cobra Venom and Its Purified Toxins; Plenum Press: New York, NY, USA, 1971; Volume 1, p. 21.

12. Huang, H.C. Release of slow reacting substance from the guinea-pig lung by phospholipases A2 of Vipera russelli snake venom. Toxicon 1984, 22, 359-372.

13. Huang, H.C.; Lee, C.Y. Isolation and pharmacological properties of phospholipases A2 from Vipera russelli (russell's viper) snake venom. Toxicon 1984, 22, 207-217.

14. Dutta, A.; Deshpande, S.B. Indian red scorpion venom-induced augmentation of cardio-respiratory reflexes and pulmonary edema involve the release of histamine. Toxicon 2011, 57, 193-198.

15. Liu, T.; Bai, Z.T.; Pang, X.Y.; Chai, Z.F.; Jiang, F.; Ji, Y.H. Degranulation of mast cells and histamine release involved in rat pain-related behaviors and edema induced by scorpion Buthus martensi karch venom. Eur. J. Pharmacol. 2007, 575, 46-56.

16. Galajda, Z.; Balla, J.; Szentmiklosi, A.J.; Biro, T.; Czifra, G.; Dobrosi, N.; Cseppento, A.; Patonay, L.; Roszer, T.; Balla, G.; et al. Histamine and H1-histamine receptors faster venous circulation. J. Cell. Mol. Med. 2011, 15, 2614-2623.

17. Roy, P.D.; Moran, D.M.; Bryant, V.; Stevenson, R.; Stanworth, D.R. Further studies on histamine release from rat mast cells in vitro induced by peptides. Characteristics of a synthetic intermediate with potent releasing activity. Biochem J. 1980, 191, 233-237.

18. Gwee, M.C.; Nirthanan, S.; Khoo, H.E.; Gopalakrishnakone, P.; Kini, R.M.; Cheah, L.S. Autonomic effects of some scorpion venoms and toxins. Clin. Exp. Pharmacol. Physiol. 2002, 29, 795-801.

19. Vasconcelos, F.; Lanchote, V.L.; Bendhack, L.M.; Giglio, J.R.; Sampaio, S.V.; Arantes, E.C. Effects of voltage-gated $\mathrm{Na}+$ channel toxins from Tityus serrulatus venom on rat arterial blood pressure and plasma catecholamines. Comp. Biochem. Physiol. C Toxicol. Pharmacol. 2005, 141, 85-92.

20. Rodriguez, A.; Zerpa, H.; Ruiz, A.; Bermudez, V.; Garcia, F.; Silva, A.; Gutierrez, L.; Villasmil, S. Effect of clonidine in mice injected with Tityus discrepans scorpion venom. Toxicon 2013, 63, 70-77. 
21. Bawaskar, H.S.; Bawaskar, P.H. Efficacy and safety of scorpion antivenom plus prazosin compared with prazosin alone for venomous scorpion (Mesobuthus tamulus) sting: Randomised open label clinical trial. BMJ 2011, 342, c7136.

22. Shore, P.A.; Burkhalter, A.; Cohn, V.H., Jr. A method for the fluorometric assay of histamine in tissues. J. Pharmacol. Exp. Ther. 1959, 127, 182-186.

23. Sagi-Eisenberg, R.; Ben-Neriah, Z.; Pecht, I.; Terry, S.; Blumberg, S. Structure-activity relationship in the mast cell degranulating capacity of neurotensin fragments. Neuropharmacology 1983, 22, 197-201.

(C) 2013 by the authors; licensee MDPI, Basel, Switzerland. This article is an open access article distributed under the terms and conditions of the Creative Commons Attribution license (http://creativecommons.org/licenses/by/3.0/). 